\title{
Changes in serum metabolites in response to ingested colostrum and milk in neonatal calves, measured by nuclear magnetic resonance-based metabolomics analysis
}

\author{
X. W. Zhao, Y. X. Qi, D. W. Huang, X. C. Pan, G. L. Cheng, H. L. Zhao, and Y. X. Yang ${ }^{1}$ \\ Institute of Animal Science and Veterinary Medicine, Anhui Academy of Agricultural Sciences, Hefei, 230031, China
}

\begin{abstract}
Uptake of colostrum is of central importance for establishing a passive immunity transfer in neonatal calves. Studies of absorption and transmission of colostral immunoglobulins have been widely reported; however, changes in the serum in response to the absorption of colostral components in neonatal calves have not been completely characterized. Here, a nuclear magnetic resonance-based metabolomics approach was used to investigate the changes in metabolites in ingested colostrum, milk, and serum after neonatal calves were fed colostrum or milk. Twenty-seven neonatal male Holstein calves were assigned to 1 of the following groups: (1) calves not fed colostrum or milk and slaughtered approximately $2 \mathrm{~h}$ after birth (control group, $\mathrm{n}$ $=6),(2)$ calves fed colostrum at 1 to $2 \mathrm{~h}$ after birth and slaughtered $8 \mathrm{~h}$ after birth $(\mathrm{n}=6),(3)$ calves fed 2 colostrum meals (at 1-2 and 10-12 h after birth) and slaughtered $24 \mathrm{~h}$ after birth $(\mathrm{n}=6),(4)$ calves fed 3 colostrum meals (at 1-2, 10-12, and 22-24 h after birth) and slaughtered $36 \mathrm{~h}$ after birth $(\mathrm{n}=6)$, or $(5)$ calves fed 2 milk meals (1-2 and 10-12 h after birth) and slaughtered $24 \mathrm{~h}$ after birth $(\mathrm{n}=3)$. Concentrations of valine, leucine, lactate, lysine, and isoleucine were higher and concentrations of lactose were lower in the groups fed colostrum and milk compared with groups not fed colostrum and milk, respectively. Metabolite changes between groups fed or not fed colostrum and milk were similar and may reflect the primary metabolic requirements of ingestion by the small intestine of neonatal calves. Concentrations of serum metabolites choline, valine, leucine, and glutamate were higher in the serum of calves that received colostrum compared with control calves. Furthermore, concentrations of serum phenylalanine, valine, and glutamate were significantly higher, whereas serum concentrations of citrate and very low density lipoproteins were lower in calves that received colostrum compared with calves
\end{abstract}

Received December 14, 2017.

Accepted March 12, 2018.

${ }^{1}$ Corresponding author: yyongxin@yahoo.com fed milk. Our results indicate that concentrations of leucine, valine, and glutamate, which were higher in the calves that ingested colostrum, may transfer into the bloodstream, and that these metabolites are associated with health benefits in the neonatal calves that received colostrum. These findings provide novel information to help us understand the mechanism by which colostrum components are metabolized and absorbed in the small intestine and then transferred into bloodstream of neonatal calves.

Key words: serum, colostrum, intestine, metabolomics

\section{INTRODUCTION}

Colostrum contains many bioactive and nutritional components and is a unique diet for neonates. Most importantly, colostral immunoglobulins play a central role in neonatal survival, as transmission into the bloodstream establishes a passive immunity transfer within the first $24 \mathrm{~h}$ of life (Quigley, 2004; Osaka et al., 2014). For this reason, many studies have investigated colostral and blood immunoglobulins and the relationship between them (Stott et al., 1979a,b; Osaka et al., 2014). In addition to establishing a passive immunity transfer, colostrum intake plays a role in stimulating intestinal mucosal growth and function as well as in enhancing the absorptive capacity of the gastrointestinal tract (Hammon et al., 2013; Steinhoff-Wagner et al., 2014). As a result, studies have found that glucose and galactose originating from lactose and xylose are absorbed in the small intestine and transferred into the bloodstream (Girard, 1990; Scharrer and Grenacher, 2000; Steinhoff-Wagner et al., 2011a). However, increases in concentrations of several growth factors, such as IGF-I and IGF-II, and insulin in the blood of calves depended on the timing of first colostrum feeding (Hammon et al., 2000), whereas absorption of colostral growth factors was barely observed in the intestine (Hammon and Blum, 1997; Ontsouka et al., 2004a). These results suggest that colostrum feeding in neonates does not only provide abundant bioactive substances and nutrition, but also contributes to improving the intestinal function for the absorption of specific colostral components. 
Although colostrum digested in the small intestine of neonatal calves has received little attention, studies on changes in the serum of neonatal calves have been widely reported. In previous studies, increases in concentrations of several free EAA and decreases in glutamine concentrations were observed in serum after calves received colostrum (Hammon and Blum, 1999; Zanker et al., 2000). Additionally, concentrations of essential fatty acids, carotene, retinol, and $\alpha$-tocopherol were significantly higher in serum of calves fed colostrum on $\mathrm{d} 1$ postcalving compared with delayed colostrum-fed calves (Blum et al., 1997). These results provide useful information for understanding the effects of colostrum intake on blood components and the potential health benefits for neonatal calves. It is notable that colostrum is a significant source of metabolites, as several metabolites can be used as potential bioactive compounds (Sundekilde et al., 2013). However, changes in blood metabolites related to colostrum intake have not been fully elucidated in neonatal ruminants.

Metabolomics, an omics field in systems biology, is widely used to investigate small metabolites within biological samples (Putri et al., 2013). As nuclear magnetic resonance (NMR) spectroscopy is a powerful method that provides direct relationships between metabolite contents and resonances, NMR approaches have been widely used to investigate the changes in metabolites of milk or serum, as well as correlations between milk and serum metabolites in dairy animals in recent years (Sundekilde et al., 2011; Klein et al., 2012; Palma et al., 2016). The results of such studies indicate that NMRbased metabolomics techniques provide a powerful tool for exploration of the effects of colostrum intake on serum metabolites in neonatal calves.

The objective of our study was to use an NMR approach to investigate the changes in serum metabolites in response to the absorption of colostrum or milk in the small intestine of neonatal calves, and to explore the absorption of metabolites into the bloodstream. We hypothesized that colostrum could be absorbed in the gastrointestinal tract and that a wide range of small molecules would transfer into the bloodstream of the calves fed colostrum. We expected our findings would provide novel information for a better understanding of the changes in serum metabolites corresponding to colostrum uptake and digestion in the gastrointestinal tract.

\section{MATERIALS AND METHODS}

\section{Animals and Experimental Procedures}

Twenty-seven neonatal male Chinese Holstein calves from the Chuzhou dairy farm (Chuzhou, China), with birth weight of $40 \pm 2 \mathrm{~kg}( \pm \mathrm{SD})$, were used in this study (Supplemental Figure S1; https://doi.org/10 $.3168 /$ jds.2017-14287). Animal care and use procedures were approved by the Animal Care Advisory Committee of the Anhui Academy of Agricultural Sciences. After birth, calves were separated from their mothers to prevent them from directly suckling colostrum. The first 2 milking colostrum samples were taken from healthy, multiparous Holstein dairy cows and pooled, and bulk milk from a healthy herd of experimental dairy farm cows was collected, placed in plastic bottles, and then stored at $-20^{\circ} \mathrm{C}$. Before use, the colostrum (not ingested colostrum) or milk (not ingested milk) was incubated in a water bath at $40^{\circ} \mathrm{C}$ and provided at approximately $8.0 \%$ of $\mathrm{BW}$ for each calf via a stomach tube, as described in previous studies (Chigerwe et al., 2012; Conneely et al., 2014). Six calves were not fed colostrum or milk and were slaughtered approximately $2 \mathrm{~h}$ after birth. These 6 calves constituted the control (Ct) group. Six calves received 1 colostrum meal at 1 to $2 \mathrm{~h}$ after birth and were slaughtered approximately $8 \mathrm{~h}$ after birth (CI group). Six calves received 2 colostrum meals at 1 to 2 and 10 to $12 \mathrm{~h}$ after birth and were slaughtered approximately $24 \mathrm{~h}$ after birth (CII group). Six calves received 3 colostrum meals at 1 to 2,10 to 12 , and 22 to $24 \mathrm{~h}$ postpartum and were slaughtered approximately $36 \mathrm{~h}$ after birth (CIII group). Three calves were fed milk instead of colostrum after birth, with 2 milk meals at 1 to 2 and 10 to $12 \mathrm{~h}$ after birth; milk-fed calves were slaughtered approximately $24 \mathrm{~h}$ after birth (M group). Samples of colostrum and milk were frozen and transferred to the laboratory.

Blood samples were collected from the jugular vein of individual calf in all groups before the calves were slaughtered. Samples were stored overnight at room temperature and centrifuged at 3,000 $\times g$ for $15 \mathrm{~min}$ at $4^{\circ} \mathrm{C}$. Supernatant was collected and aliquoted into $1.5-\mathrm{mL}$ tubes, then frozen at $-20^{\circ} \mathrm{C}$. After the blood samples were collected, the calves were slaughtered. Mid-duodenum, -jejunum, and -ileum segments were separated within 30 min after opening the abdominal cavity (Liang et al., 2014). Ingested colostrum or milk was collected from mid-jejunum segments, frozen in liquid nitrogen, and then stored at $-80^{\circ} \mathrm{C}$ until analysis.

\section{NMR Spectroscopy Analysis}

Colostrum and milk samples were thawed at room temperature and centrifuged at $4,000 \times g$ for $30 \mathrm{~min}$ at $4^{\circ} \mathrm{C}$. The fat layer was removed and skim milk was collected. A total of $500 \mu \mathrm{L}$ of skim milk from each sample was mixed with $1.0 \mathrm{~mL}$ of methanol and $500 \mu \mathrm{L}$ of double-distilled $\mathrm{H}_{2} \mathrm{O}$, followed by periodical vortexing. The mixture was centrifuged at $12,000 \times g$ for 10 
min at $4^{\circ} \mathrm{C}$. The supernatant was collected and centrifuged to dry with a Speed Vac Concentrator (SPD121P, Thermo Savant, Waltham, MA). Dried samples were dissolved with $600 \mu \mathrm{L}$ of solution $\left(0.1 \mathrm{M} \mathrm{NaH}_{2} \mathrm{PO}_{4} /\right.$ $\mathrm{K}_{2} \mathrm{HPO}_{4}$ and $0.1 \% \mathrm{NaN}_{3}$ in deuterium oxide, $\mathrm{pH}$ 7.4), followed by periodical vortexing. To analyze serum samples, $200 \mu \mathrm{L}$ of thawed serum was mixed with 400 $\mu \mathrm{L}$ of solution $\left(0.045 \mathrm{M} \mathrm{NaH} \mathrm{PO}_{4} / \mathrm{K}_{2} \mathrm{HPO}_{4}\right.$ and $0.1 \%$ $\mathrm{NaN}_{3}$ in deuterium oxide, pH7.4). The mixtures were centrifuged at $16,000 \times \mathrm{g}$ for $10 \mathrm{~min}$ at $4^{\circ} \mathrm{C}$, and 550 $\mu \mathrm{L}$ of supernatant from each sample was collected and placed into a 5-mm NMR tube. Two replicates of each sample were prepared for NMR spectroscopy.

The NMR scans were performed at a temperature of $298 \mathrm{~K}$, with a proton frequency of $599.83 \mathrm{MHz}$, using an Agilent DD2600 MHz spectrometer (Agilent Technologies, Santa Clara, CA). The one-dimensional nuclear Overhauser enhancement spectroscopy preset sequence for water resonance was selected. A $90^{\circ}$ pulse length with a relaxation delay of $10 \mu \mathrm{s}$ and an acquisition time of $1.36 \mathrm{~s}$ were applied, and a total of 32,000 data points were collected, with 64 scans and a spectral width of $20 \mathrm{ppm}$. Prior to applying the Fourier transformation, free-induction decays of the collected ${ }^{1} \mathrm{H}-\mathrm{NMR}$ spectra were extended using a line-broadening factor of $1.0 \mathrm{~Hz}$ to improve the signal-to-noise ratio. The NMR spectra were manually phase- and baseline-corrected, and all were referenced to sodium trimethylsilyl-[2,2,3,32H4]-1-propionate (Merck, Darmstadt Germany) and evaluated for the individual metabolite signals. For the signals of the milk samples, the spectra were segmented into integral regions of chemical shifts $(\boldsymbol{\delta}) 9.5$ to 0.6 with a width of $0.002 \mathrm{ppm}$ for each segment, and water resonance $(\delta 5.20-4.72 \mathrm{ppm})$ and buffer resonance $(\delta$ $3.327-3.40 \mathrm{ppm}$ ) regions were removed. For the signals of the serum samples, the spectra were segmented into integral regions $\delta 9.5$ to 0.5 with a width of $0.002 \mathrm{ppm}$ for each segment, and water resonance $(\delta$ 5.20-4.20 $\mathrm{ppm})$ and urea resonance $(\delta 6.20-5.50 \mathrm{ppm})$ regions were removed.

\section{Multivariate Statistical Analysis}

The intensity of each peak signal in the NMR spectra was proportional to the concentration in the samples. The NMR data were normalized and transferred to the SIMCA-P ${ }^{+} 13.0$ software package (Umetrics AB, Umeå, Sweden) for multivariate statistical analysis. To investigate the changes in metabolites of colostrum and milk, colostrum metabolites were compared with the ingested colostrum metabolites from the CI, CII, and CIII groups; milk metabolites were compared with the ingested milk from $M$ group. In the serum analysis, metabolites from Ct group were compared with CI, CII, and CIII, as well as M groups, respectively. In addition, metabolites of ingested colostrum or milk and serum were also compared between CII and M groups. Principal component analysis (PCA) was applied to test clustering profiles and trends among the studied samples. Supervised partial least squares discriminant analysis (PLS-DA) and orthogonal partial least squares discriminant analysis (OPLS-DA) procedures were performed on the unit variance scaling. Three parameters, including $R^{2} X$ and $R^{2} Y$ (to assess the model quality) and $\mathrm{Q}^{2}$ (to measure the model predictive ability), were used to evaluate the quality and confidence of the OPLS-DA models, calculated by cross-validation procedures. Of them, $R^{2} X$ and $R^{2} Y$ were used to assess the model quality, and $\mathrm{Q}^{2}$ was used to measure the model predictive ability.

Score plots of the OPLS-DA model were used to visualize the separation between the studied groups, and coefficient loading plots were used to examine the spectral variables contributing to sample differentiation on the score plot. The colors of spectral signals projected onto the coefficient plots are proportional to the intensity of metabolite signals that are used to distinguish between 2 studied groups, in which red coloring indicates a high correlation and blue coloring indicates no correlation. Correlation coefficients were calculated to evaluate potential associations between metabolites in the experimental groups on the basis of the Pearson product-moment regression coefficient. Relative concentrations of metabolites were subjected to the nonparametric Mann-Whitney $U$ test used to assess statistically significant differences between the 2 groups. Differential metabolites between the 2 groups were assigned by comparison with signals of chemical shifts in the Human Metabolome Database (www.hmdb .ca).

\section{RESULTS}

\section{Differences in Metabolites in Calves Fed or Not Fed Colostrum and Milk}

Representative ${ }^{1} \mathrm{H}-\mathrm{NMR}$ spectra $\left(\begin{array}{ll}\delta & 0.5-5.4 \text { and } \delta\end{array}\right.$ 5.4-9.5) of groups not fed colostrum and milk and fed colostrum and milk are shown in Supplemental Figure S2 (https://doi.org/10.3168/jds.2017-14287). Several signals from lactose and other metabolites, such as carnitine, creatinine, and citrate, were detected. A PCA approach was used to present the clustering patterns of samples based on the mean-centered NMR spectra data. The PCA score plots from groups not fed colostrum and milk and fed colostrum and milk are shown 
in Supplemental Figure S3 (https://doi.org/10.3168/jds .2017-14287). Metabolite profiles between calves not fed colostrum and intestinal colostrum of CI, CII, and CIII groups as well as between calves not fed milk and fed milk were clearly different. The ellipse in the score plots defines the Hotelling's $\mathrm{T}^{2}$ confidence region and provides a 95\% confidence interval for the observed samples.

The supervised OPLS-DA approach was used to evaluate the different metabolites in the small intestine in groups not fed and fed colostrum and milk. The OPLSDA score plots between the studied groups were well separated without any overlap. Data originated from 1 predictive component and 2 orthogonal components, in which $\mathrm{Q}^{2}$ values were at least 0.832 , indicating that the models were effective for predictive reliability. Differences in metabolites between colostrum and milk and samples from CI, CII, CIII, and M groups identified using OPLS-DA score plots and corresponding coefficient loading plots are shown in Figure 1. The differential metabolites based on the signals of chemical shift are listed in Table 1 and Supplemental Table S1 (https:// doi.org/10.3168/jds.2017-14287).

Based on the results of the NMR analysis, we found lower concentrations of lactose and citrate and higher concentrations of valine, leucine, isoleucine, and creatine in colostrum compared with milk. Compared with groups not fed colostrum, concentrations of several metabolites, such as lactate, succinate, and glutamate, were significantly higher in the CI, CII, and CIII groups, whereas concentrations of lactose and uridine diphosphate were significantly lower. Compared with groups not fed milk, concentrations of several metabolites, such as inosine, succinate, and hypoxanthine, were higher whereas concentrations of lactose and citrate were lower in calves in the $\mathrm{M}$ group. Although many differences were observed between the metabolites in milk and colostrum, changes in metabolites from colostrum to ingested colostrum and from milk to ingested milk were unexpectedly very similar after calves were fed colostrum and milk. However, concentrations of 5 metabolites were different in the ingested colostrum and milk approximately $24 \mathrm{~h}$ after birth; of them, inosine, uridine diphosphate, hypoxanthine, and 2 unknown metabolites were higher in the ingested milk.

\section{Differences Between Serum Metabolites in Calves Not Fed or Fed Colostrum and Fed Milk}

Representative NMR spectra $(\delta 0.5-5.4$ and $\delta 5.4-9.0)$ of the serum from calves in the Ct group, CI, CII, and CIII groups, and calves in the M group are shown in Supplemental Figure S4 (https://doi.org/10.3168/jds .2017-14287). The NMR spectra data were subjected to PCA analysis to provide the clustering patterns of the studied samples. The PCA score plots are shown in Supplemental Figure S5 (https://doi.org/10.3168/jds .2017-14287), which clearly shows that the metabolite profiles of serum of Ct calves and CI, CII, and CIII calves were different. Similarly, metabolite profiles of serum in calves from CII and M groups were clearly different.

The supervised OPLS-DA approach was also used to investigate the differential metabolites in serum between the different treatment groups. Differences in serum metabolites of calves from the control and experimental groups obtained using OPLS-DA score plots and corresponding coefficient loading plots are shown in Figure 2. Models of 1 predictive component and 2 orthogonal components were present, in which $\mathrm{Q}^{2}$ values were at least 0.817 , indicating robust predictive reliability. The identified serum metabolites in the different treatment groups are listed in Table 2 and Supplemental Table S2 (https://doi.org/10.3168/jds.2017-14287).

According to the results of OPLS-DA analysis, concentrations of metabolites of glutamate, glycerophosphocholine/phosphocholine, lipid, choline, and tyrosine were significantly higher in serum of calves from the CI, CII, and CIII groups, whereas concentrations of glycerol and an unknown metabolite were lower compared with the Ct group. In addition, concentrations of valine, leucine, and histidine were higher, whereas concentrations of creatinine, citrate, and glutamine were lower in serum of calves in the CII and CIII groups compared with calves in the $\mathrm{Ct}$ group. However, in the M group, serum concentrations of pyruvate and histidine were higher whereas concentrations of glycerol, allantoin, guanine, and citrate were lower compared with calves in the $\mathrm{Ct}$ group. We also found that changes in serum metabolites in calves fed colostrum (CII group) and milk (M group) had a similar pattern, and only 7 serum metabolites were significantly different. Of these, concentrations of valine, glutamate, and creatine were higher, whereas concentrations of citrate and $\mathrm{N}$-acetyl glycoprotein signals were lower in calves fed colostrum (CII group) compared with the calves fed milk.

\section{Relationships Between Metabolites in Ingested Colostrum and Serum Metabolites After Ingestion of Colostrum}

Concentrations of leucine, valine, isoleucine, glutamate, lysine, tyrosine, lactate, and succinate were higher in the ingested colostrum collected from the gastrointestinal tract than in colostrum that was not ingested, whereas concentrations of lactose, choline, and uridine diphosphate were lower in the ingested co- 

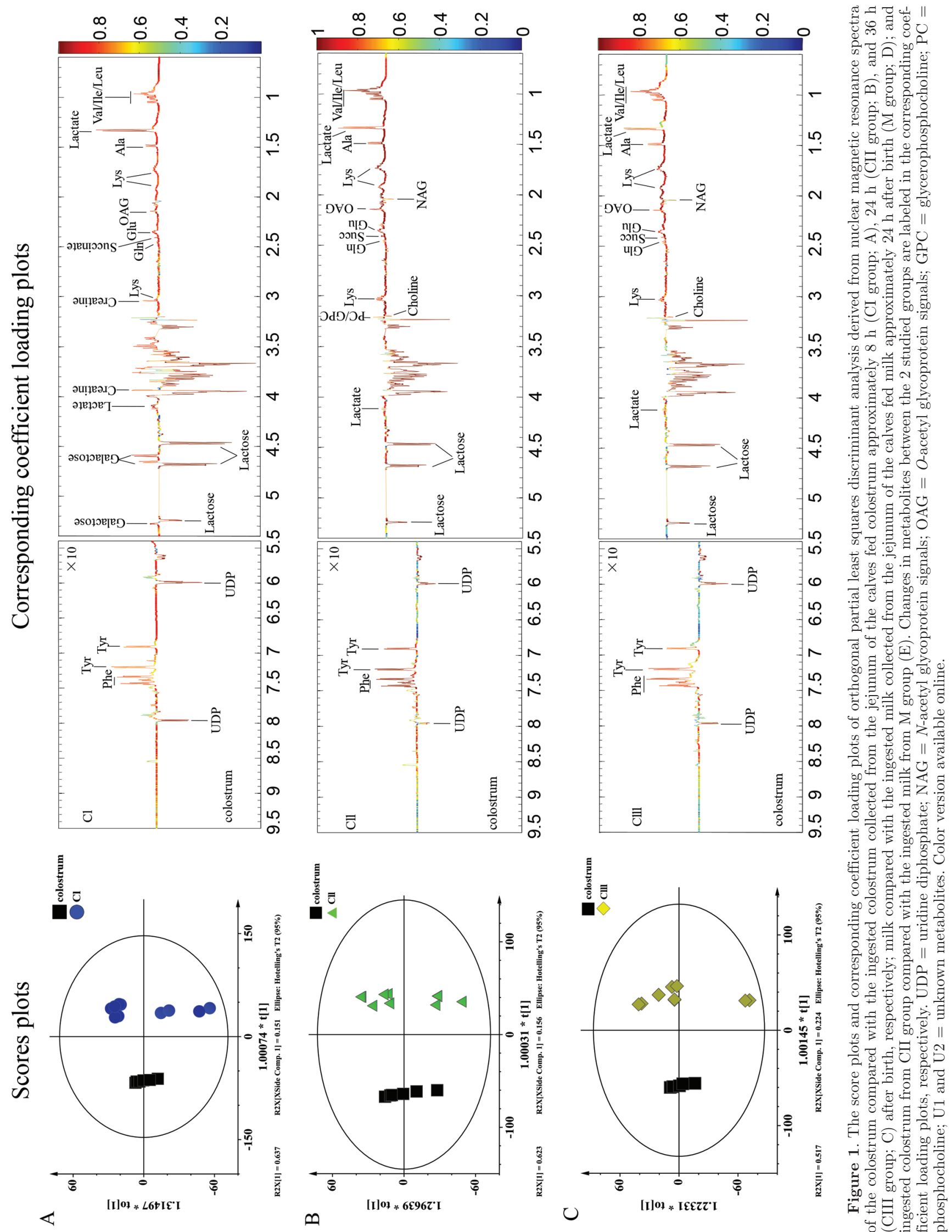

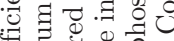

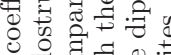

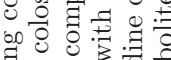

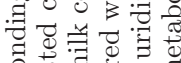

요의

क⿺辶大: 产 0

可

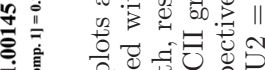

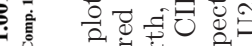

竞

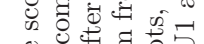

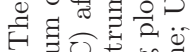

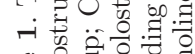

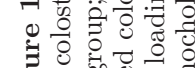

5000

setent 

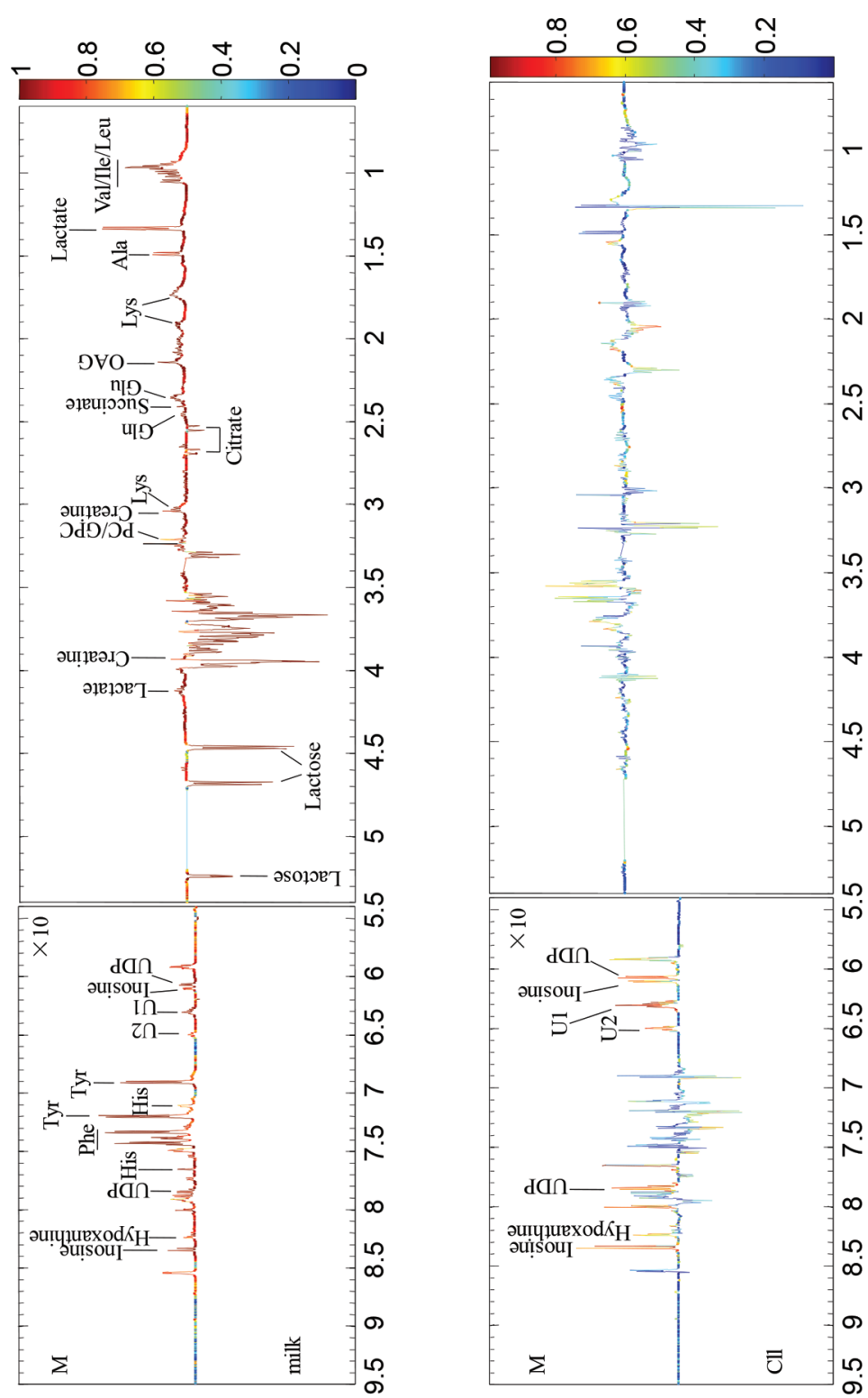

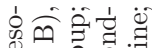

40 응응

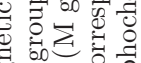

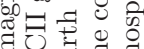

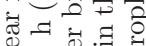

政要

द्य

घ

$\exists=0$

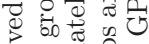

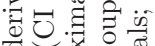

$0=00$

$\infty$ 娄的

넘

政

สี.

a

प्रे के एँ

㕩

क 50

때용

낭 $: \because 11$

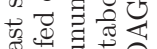

吸

(1)

获

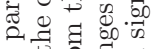

తే

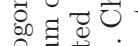

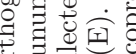

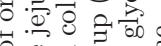

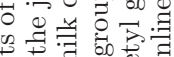

음

bo

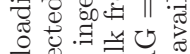

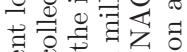

$\overrightarrow{0}: \vec{q}$

E 50

की $0 . \exists$ का

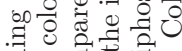

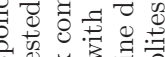

年

.
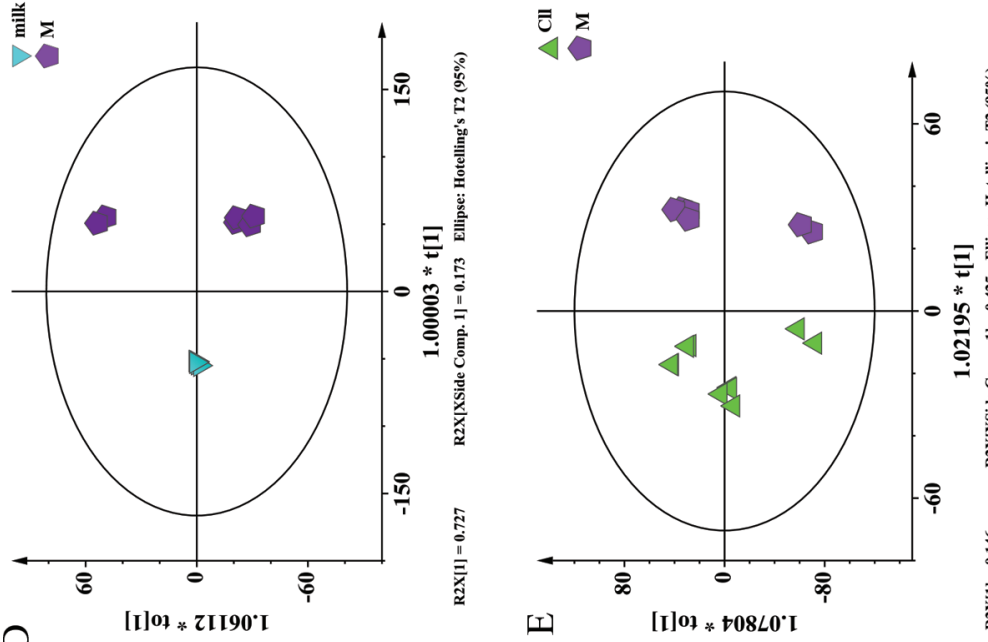

8 os

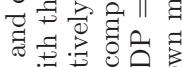

落势

a d क्ष

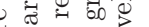

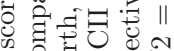

0 영

स 寻 总

के

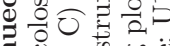

s.

要

0

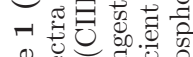

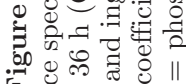

II

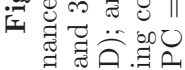


ZHAO ET AL.

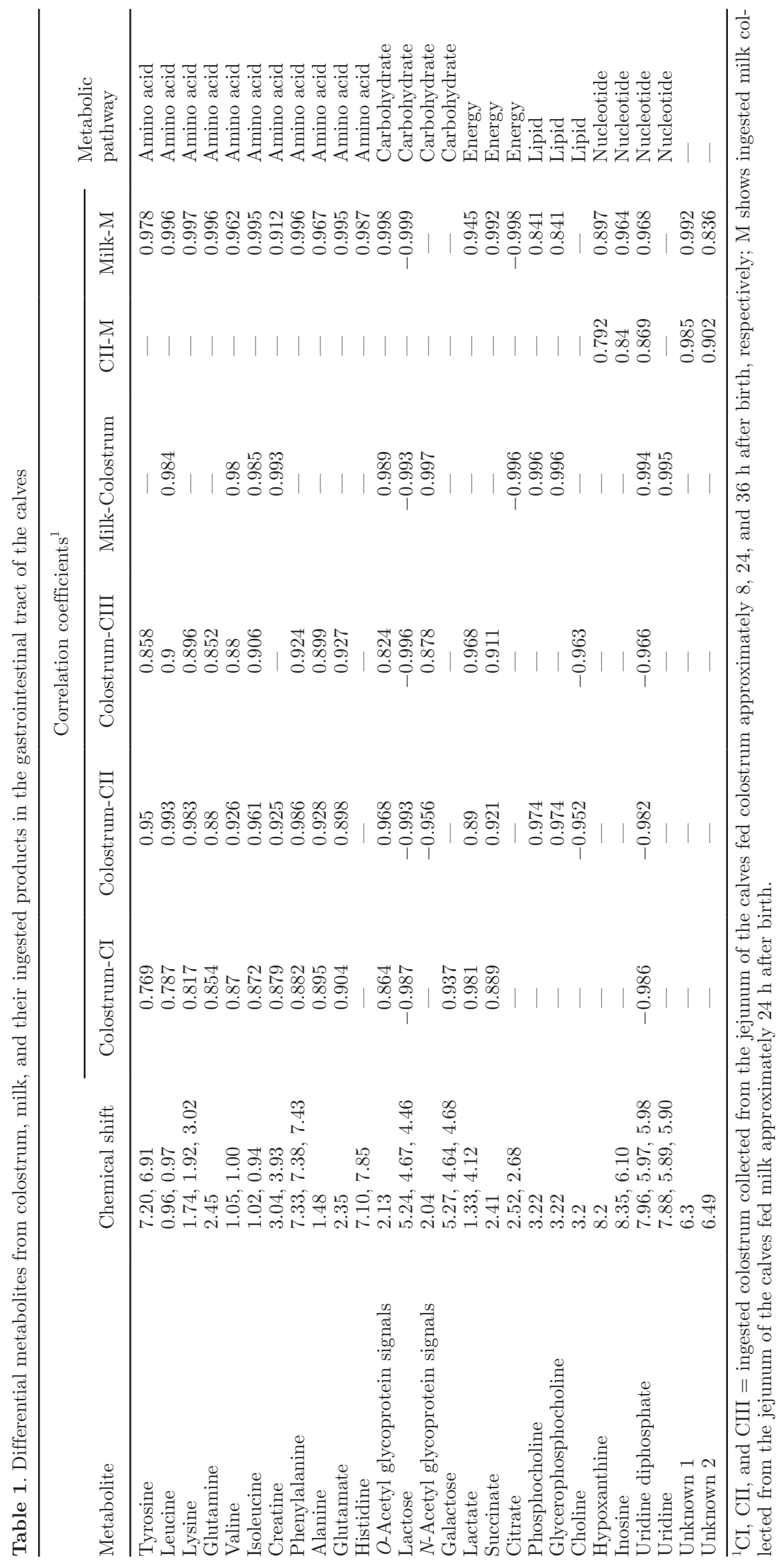


lostrum. Accordingly, concentrations of choline, lipid, glycerophosphocholine/phosphocholine, tyrosine, and glutamate were higher in the serum of the calves in CI, CII, and CIII than in Ct calves. On the basis of changes in metabolites in the ingested colostrum and serum, several metabolites from the ingested colostrum, such as tyrosine and glutamate, were clearly positively associated with increased concentrations of these metabolites in serum.

\section{DISCUSSION}

Several previous studies indicated that bovine colostrum is a significant source of metabolites, with higher concentrations of AA, carbohydrates, lipids, and vitamins than milk (Gopal and Gill, 2000; Kamada et al., 2007; McGrath et al., 2016). In accordance with these results, we found that concentrations of valine, leucine, creatine, and uridine were higher, whereas concentrations of lactose were lower in colostrum as compared with milk, determined by the NMR-based metabolomics in the current study. Similarly, in sows, concentrations of several metabolites, such as citric acid, glycerophosphocholine, creatine, and choline were higher in colostrum than in milk, whereas concentrations of betaine, phosphocholine, and taurine were lower in colostrum than in milk based on NMR approaches (Curtasu et al., 2016). Studies of human breastmilk metabolites found that concentrations of alanine, glutamate, valine, and choline were higher in colostrum than in milk (Spevacek et al., 2015; Wu et al., 2016). Taken together, these studies suggest that colostrum is rich in some metabolites that may contribute to meeting the specific requirements of newborns compared with milk.

In neonatal calves, colostrum ingested in the gastrointestinal tract is used to meet the absorption requirements of the small intestine. We found that several metabolites associated with carbohydrates, AA, lipids, and nucleotides were altered in the ingested colostrum compared with colostrum not ingested. For example, concentrations of lactose were lower in the ingested colostrum because the lactase activity in the small intestine increased after neonates were fed with colostrum, which served as a primary determinant of lactose digestion (Burrin et al., 1994; Kelly and Coutts, 2000; Ontsouka et al., 2004b). As a result, lactose was digested as glucose and galactose, and these were absorbed in the small intestine to meet the energy demands of neonates after birth (Girard, 1990; Steinhoff-Wagner et al., 2014). In accordance with results from previous studies, we found significantly lower lactose concentrations in the ingested colostrum from CI, CII, and CIII groups compared with colostrum not ingested. Similarly, we also found that lactose concentrations in the ingested milk from calves in the $\mathrm{M}$ group were lower than in milk not ingested. In addition, lactose concentrations were not different between the ingested colostrum from calves in the CII group and the ingested milk from calves in the M group, although lactose concentrations were significantly lower in colostrum than in milk. Interestingly, lactose in human breastmilk has been considered an inducer of innate immunity (Cederlund et al., 2013). According to the changes in AA, we found higher concentrations of valine, leucine, glutamate, glutamine, and lysine in the ingested colostrum from calves in the CI, CII, and CIII groups compared with colostrum not ingested. A previous study found that concentrations of total EAA were relatively low in colostrum, and several AA, such as threonine, arginine, and glutamine, were not detected in colostrum (Zanker et al., 2000). Subsequently, a study of colostrum passing through the stomach and gut of piglets indicated that caseins and $\beta$-LG are likely to be digested when they reach the small intestine, based on proteomics analysis (Danielsen et al., 2011). Based on our results and the discussion of previous studies, we concluded that several types of colostrum components were digested in the gastrointestinal tract, resulting in higher concentrations of succinate, valine, leucine, alanine, and lysine in the ingested colostrum. This result may reflect the primary ingested requirements of colostrum by the neonatal intestine that contributed to providing a suitable metabolite profile for the absorption by the small intestine of neonates; however, these aspects need further investigation.

As a result of feeding colostrum, glucose concentrations were significantly higher in the serum of calves. Our results were similar to the results of a previous study that found that serum glucose concentrations were increased in calves that received colostrum (Steinhoff-Wagner et al., 2011b). For neonatal calves, lactose intake is not sufficient to meet glucose demands (Girard, 1990), and endogenous glucose production and gluconeogenesis have been observed in labeled-glucose studies (Steinhoff-Wagner et al., 2011b). In contrast, glycerol concentrations were lower in the serum of calves fed colostrum. Previous studies suggested that glycerol is an important gluconeogenic substrate that is taken up by the liver and converted into glucose (Phillips et al., 2002; Steinhoff-Wagner et al., 2011b). Similarly, we found that glycerol concentrations were lower in the serum of calves fed milk compared with control calves. Unexpectedly, glucose and glycerol concentrations were not different in the serum of calves from CII and M groups; thus, we believe that serum glycerol could en- 

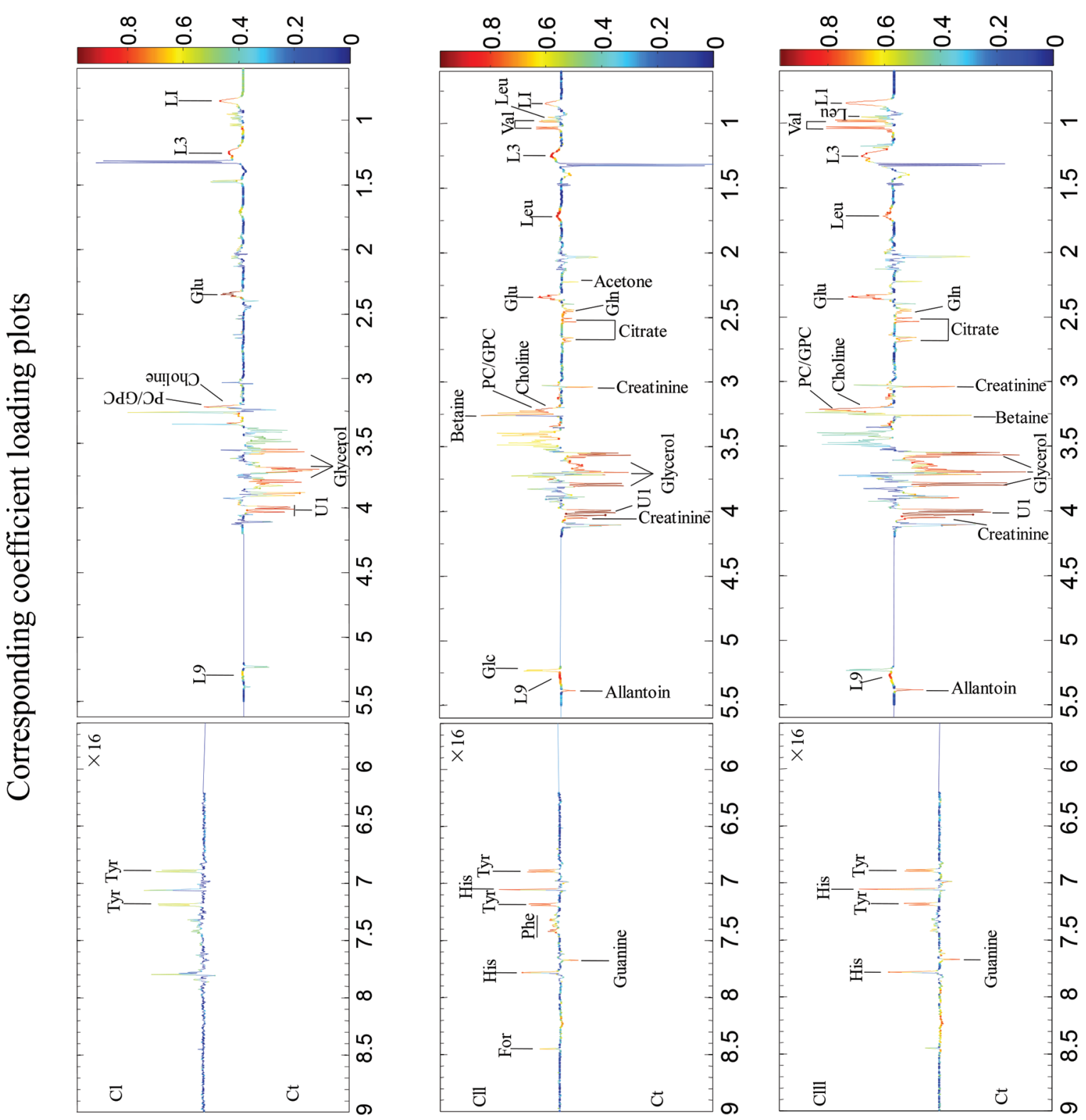

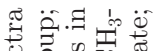

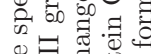

䒕司艺

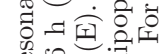

을

尊

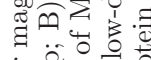

कै

菏牙

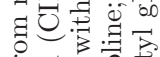

牙㣢

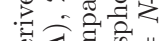

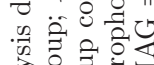

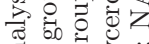

者它

젹

곤

善
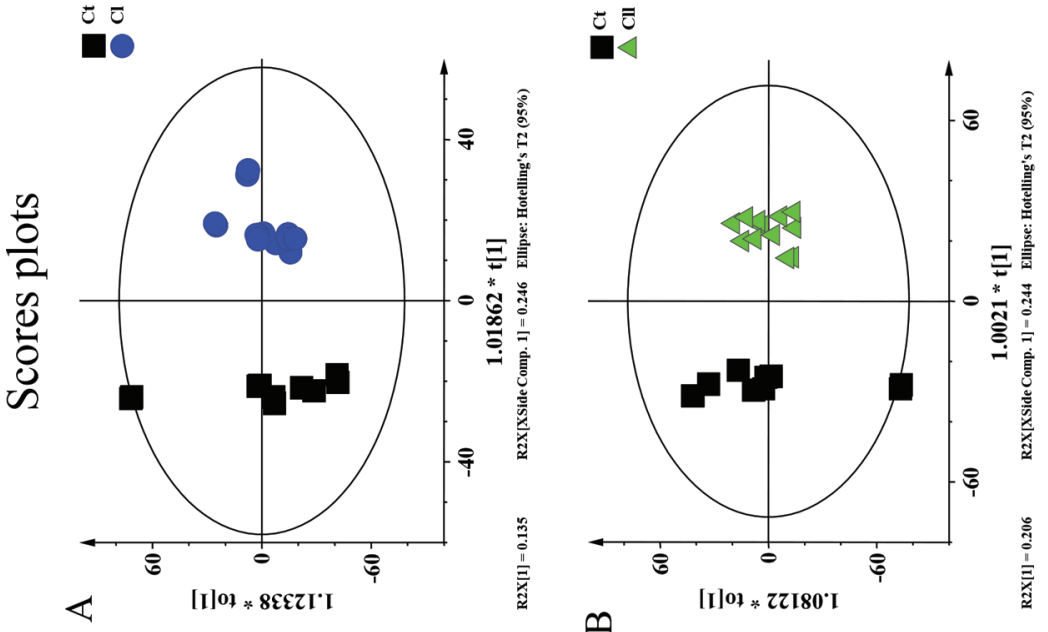

च 음

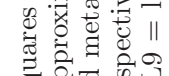

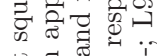

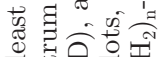

0 .

的

- 2 西

- $\quad 000$.

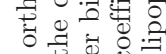

.

을

on

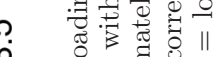

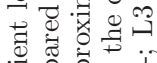

- $\quad$ d.

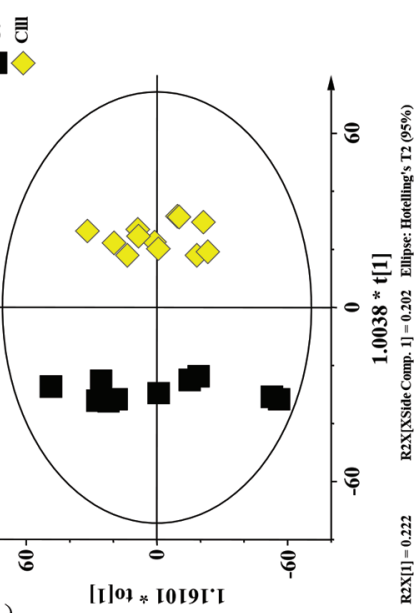

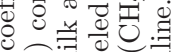

60 自骂

牙它矛过。

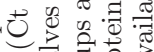

西范

o

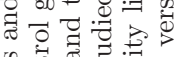

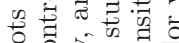

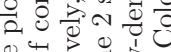

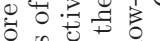

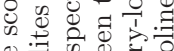

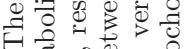

ง

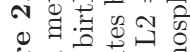

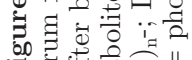

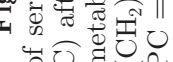



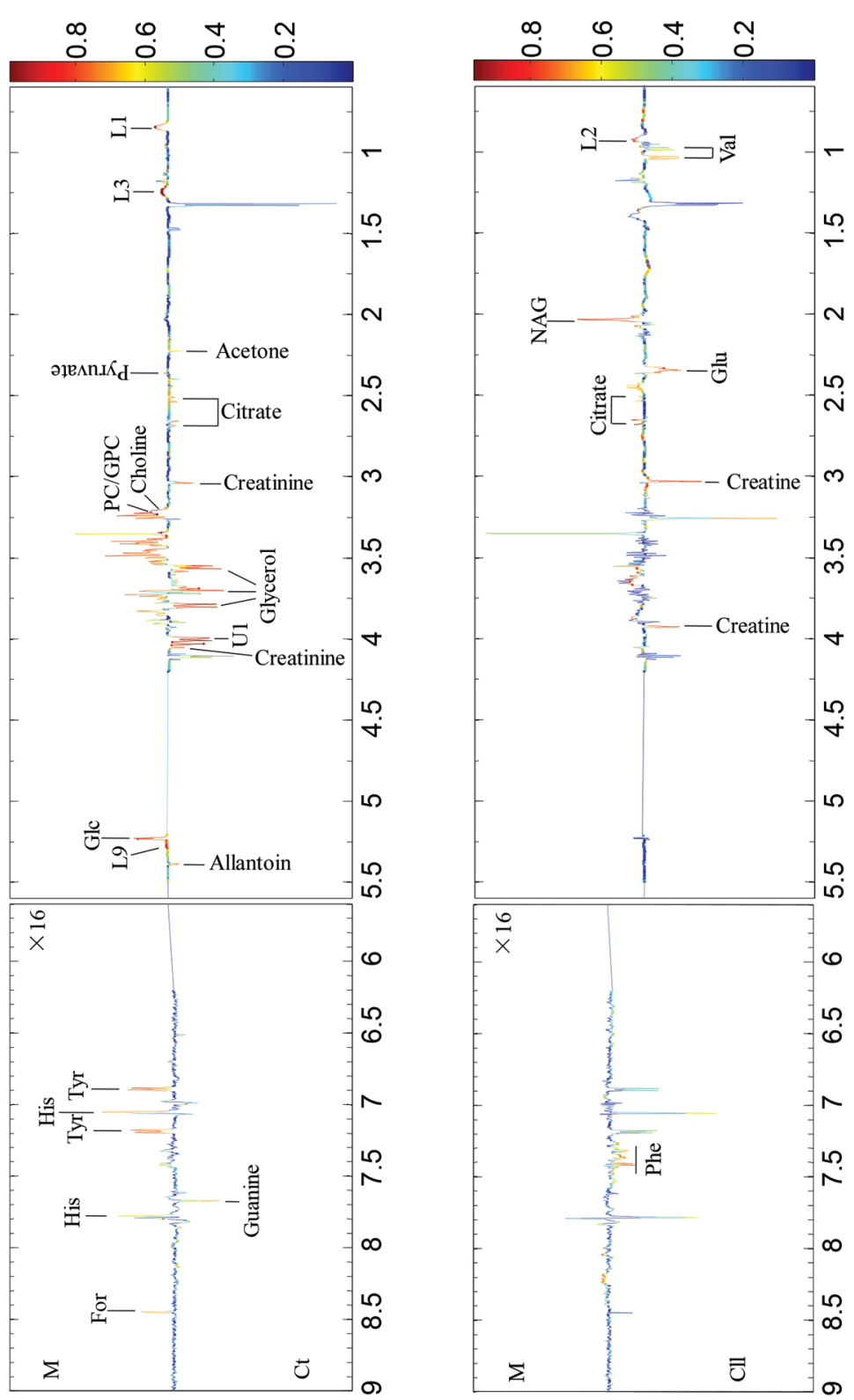

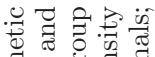

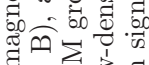

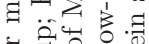

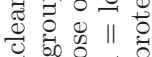

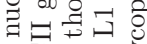

g 0 -

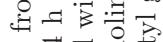

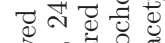

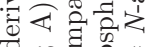

s) 웡

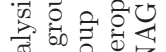

㝴娄

至

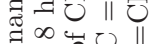

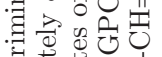

.

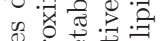

运

해월

10 㽞政

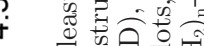

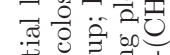

눙

幽 50 .

สే

80 듄

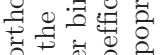

प्ति

in

웅현

00 동 क्ते

毒官要

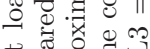

붕

붕

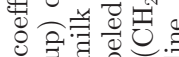

50 :

品舟

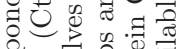

क्षे

5 o

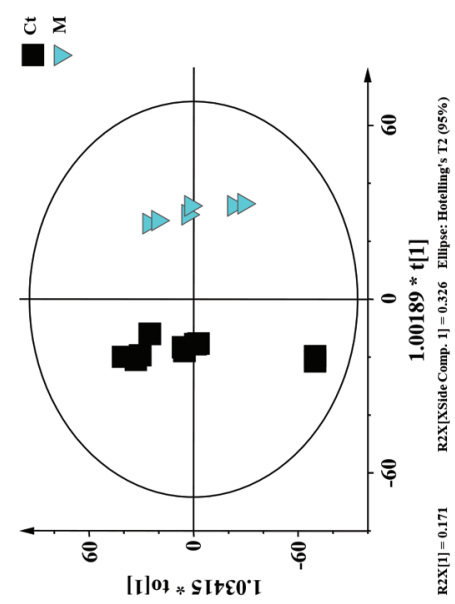

A

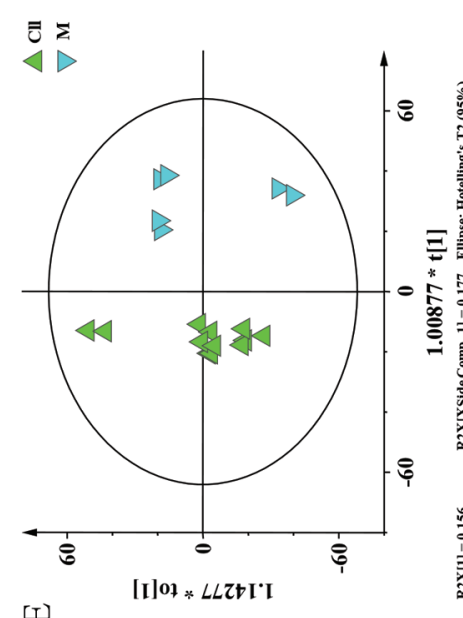

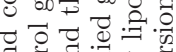

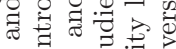

is

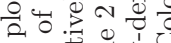

0

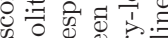

苇 0

H.

कि口

政造

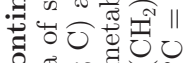

记要前

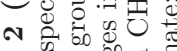

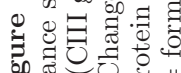

些总

Journal of Dairy Science Vol. 101 No. 8, 2018 
Table 2. Differential metabolites of serum derived from the calves not fed or fed colostrum and milk

\begin{tabular}{|c|c|c|c|c|c|c|c|}
\hline Metabolite $^{1}$ & Chemical shift & \multicolumn{5}{|c|}{ Correlation coefficients $^{2}$} & $\begin{array}{l}\text { Metabolic } \\
\text { pathway }\end{array}$ \\
\hline Glycerol & $3.56,3.68,3.77$ & -0.892 & -0.959 & -0.948 & -0.93 & - & Lipid \\
\hline Lipid, -CH=CH- & 5.27 & 0.675 & 0.876 & 0.802 & 0.829 & - & Lipid \\
\hline $\mathrm{LDL}, \mathrm{CH}_{3^{-}}\left(\mathrm{CH}_{2}\right)_{\mathrm{n}^{-}}$ & 0.84 & 0.827 & 0.864 & 0.866 & 0.93 & - & Lipid \\
\hline Phosphorylcholine & 3.22 & 0.872 & 0.747 & 0.837 & 0.922 & - & Lipid \\
\hline Formate & 8.45 & - & 0.699 & - & 0.732 & - & Lipid \\
\hline VLDL, $\mathrm{CH}_{3-}\left(\mathrm{CH}_{2}\right)_{\mathrm{n}^{-}}$ & 0.92 & - & - & - & - & 0.919 & Lipid \\
\hline Tyrosine & $6.88,7.18$ & 0.629 & 0.836 & 0.81 & 0.822 & - & Amino acid \\
\hline Glutamate & 2.35 & 0.981 & 0.871 & 0.846 & - & -0.772 & Amino acid \\
\hline Valine & $0.98,1.04$ & - & 0.802 & 0.846 & - & -0.756 & Amino acid \\
\hline Phenylalanine & $7.33,7.38,7.43$ & - & 0.842 & - & - & -0.826 & Amino acid \\
\hline Histidine & $7.06,7.78$ & - & 0.912 & 0.887 & 0.768 & - & Amino acid \\
\hline Creatinine & $3.04,4.05$ & - & -0.844 & -0.862 & -0.801 & - & Energy \\
\hline Acetone & 2.23 & - & -0.655 & - & -0.778 & - & Energy \\
\hline Pyruvate & 2.36 & - & - & - & 0.82 & - & Energy \\
\hline Creatine & $3.03,3.92$ & - & - & - & - & -0.899 & Energy \\
\hline Allantoin & 5.39 & - & -0.852 & -0.922 & -0.806 & - & Nucleotide \\
\hline Guanine & 7.67 & - & -0.794 & -0.826 & -0.714 & - & Nucleotide \\
\hline Glucose & 5.23 & - & 0.668 & - & 0.842 & - & Carbohydrate \\
\hline Unknown 3 & 4 & -0.92 & -0.977 & -0.96 & 0.972 & - & - \\
\hline
\end{tabular}

${ }^{1} \mathrm{LDL}=$ low-density lipoprotein; VLDL = very-low-density lipoprotein.

${ }^{2} \mathrm{Ct}=$ serum derived from control calves; CI, CII, and CIII = serum derived from the calves fed colostrum approximately 8,24 , and $36 \mathrm{~h}$ after birth, respectively; $\mathrm{M}=$ serum derived from the calves fed milk approximately $24 \mathrm{~h}$ after birth.

ter the gluconeogenesis pathway to meet the energy requirements of neonatal calves.

Moreover, several previous studies found that concentrations of total free EAA and several individual AA, such as lysine, methionine, threonine, and glutamate, increased in serum within $12 \mathrm{~h}$ after calves received colostrum (Hammon and Blum, 1999; Zanker et al., 2000). More recently, 2-dimensional GC-MS was used to investigate the changes in serum metabolites of neonatal calves in response to colostrum intake, and the results indicated that concentrations of several EAA, such as threonine, tryptophan, and methionine, were significantly higher in serum from calves fed colostrum and that these concentrations followed a time-dependent pattern (Miyazaki et al., 2017). In another study, compared with a baseline, postprandial plasma concentrations of isoleucine, lysine, tyrosine, and glutamine were significantly higher in calves fed a milk replacer twice daily using NMR spectroscopy (Ghaffari et al., 2017). In particular, previous studies have indicated that nearly all of the colostral components were absorbed by the neonatal small intestine (Dividich et al., 2007; Lin et al., 2009). As a result, we found that several AA were significantly higher in the serum of calves fed colostrum, whereas concentrations of glutamine were lower in the serum of calves fed colostrum than in control calves; this result was in agreement with the results of previous studies (Hammon and Blum, 1999; Zanker et al., 2000). However, serum concentrations of histidine and tyrosine were significantly higher in the calves fed milk than in control calves. More importantly, several metabolites have been implicated in multiple biological functions; for example, glutamine plays a crucial role in the metabolism of AA, carbohydrates, and lipids (Lobley et al., 2001; Roth et al., 2002). Glutamine could serve as the main fuel for newborn enterocytes and as a precursor for DNA synthesis to promote gut growth and development (Posho et al., 1994; Roth et al., 2002). Leucine, in particular, has been considered as a potent activator of glucose uptake and glycogen synthesis (Nishitani et al., 2002). Additionally, the branched-chain AA valine, leucine, and isoleucine have been implicated in promoting protein synthesis and transcription in liver and other tissues via mammalian target of rapamycin signaling pathways (D'Antona et al., 2010). Branched-chain AA have also been found to 
be involved in regulating lymphocyte growth and proliferation and natural killer cell activity (Calder, 2006; Nakamura et al., 2007). Thus, we suggest that higher concentrations of metabolites in the ingested colostrum and serum of the calves fed colostrum could contribute to meeting the requirements of calves' growth and development, as well as immunity.

Furthermore, choline concentrations were lower in the ingested colostrum, whereas choline concentrations were higher in serum of calves fed colostrum. In breastfed infants at 12 to $180 \mathrm{~d}$ after birth, serum choline concentrations were positively correlated with choline, phosphocholine, glycerophosphocholine, and total choline contents of breastmilk (Ilcol et al., 2005). In general, phosphocholine, glycerophosphocholine, and phosphatidylcholine serve as a form of storage for choline (Hollenbeck, 2012). Importantly, choline is a precursor for the biosynthesis of phospholipids (phosphatidylcholine and sphingomyelin), which are essential structural components of all cellular membranes (Michel et al., 2006; Ridgway, 2013). Choline-deficient diets resulted in altered intestinal morphology and impaired intestinal lipid metabolism in lactating rats (da Silva et al., 2015). As evident based on these studies, choline may contribute to the structural integrity of intestinal membranes and lipid transport and metabolism. In addition, a previous study found higher phospholipid and cholesterol concentrations in serum of calves that received colostrum (Blum et al., 1997). Cholesterol and lipids were absorbed from the intestine and packaged into very low density lipoproteins for transport in the bloodstream (Scow et al., 1980; Beisiegel, 1998). We also found higher concentrations of lipids in serum of calves that received colostrum. Thus, we believe that changes in the ingested colostrum contributed to improving intestinal morphology for absorption efficiency of the small intestine, and that some of these metabolites may be transported into blood. However, the relationship between the colostrum components absorption by the small intestine and transmission into the bloodstream needs further investigation.

\section{CONCLUSIONS}

Ours is the first study to characterize the effects of colostrum and its digestion in the gastrointestinal tract on serum metabolites of neonatal calves using NMR approaches. The primary metabolite characterization of colostrum and milk ingested and not ingested revealed that concentrations of several metabolites, such as lysine, valine, leucine, lactate, and isoleucine were higher in the ingested colostrum and milk compared with colostrum and milk not ingested. In addition, the metabolic profile of ingested colostrum was similar to that of ingested milk and was used to meet the requirements of neonates. Compared with concentrations of serum metabolites in control calves, concentrations of choline, tyrosine, and glutamate were higher in serum of calves that received colostrum. Concentrations of phenylalanine, valine, and glutamate were also significantly higher, whereas concentrations of citrate and very low density lipoprotein were lower in serum of calves that received colostrum compared with calves fed milk. These findings suggest that metabolites in ingested colostrum might facilitate the absorption of small molecule compounds by the small intestine of neonatal calves. Metabolomics-based NMR analysis of colostrum, ingested colostrum, and serum may provide novel information to help understand the mechanism of the absorption of colostrum components in the small intestine and contribute to understanding the serum metabolome profile of neonatal calves.

\section{ACKNOWLEDGMENTS}

This work was supported by the National Natural Science Foundation of China (No. 31572434), the Special Fund for Agro-scientific Research in the Public Interest (201403071), and Youth Innovation Fund of Anhui Academy of Agricultural Sciences (No. 16B0408). The authors thank staff of the ruminant nutrition laboratory at the Institute of Animal Science and Veterinary Medicine, Anhui Academy of Agricultural Sciences (Hefei, China), for their assistance in feeding work and pattern analysis.

\section{REFERENCES}

Beisiegel, U. 1998. Lipoprotein metabolism. Eur. Heart J. 19:A20-A23. Blum, J. W., U. Hadorn, H. P. Sallmann, and W. Schuep. 1997. Delaying colostrum intake by one day impairs plasma lipid, essential fatty acid, carotene, retinol and $\alpha$-tocopherol status in neonatal calves. J. Nutr. 127:2024-2029.

Burrin, D. G., M. A. Dudley, P. J. Reeds, R. J. Shulman, S. Perkinson, and J. Rosenberger. 1994. Feeding colostrum rapidly alters enzymatic activity and the relative isoform abundance of jejunal lactase in neonatal pigs. J. Nutr. 124:2350-2357.

Calder, P. C. 2006. Branched-chain amino acids and immunity. J. Nutr. 136:288S-293S.

Cederlund, A., Y. Kai-Larsen, G. Printz, H. Yoshio, G. Alvelius, H. Lagercrantz, R. Strömberg, H. Jörnvall, G. H. Gudmundsson, and B. Agerberth. 2013. Lactose in human breast milk an inducer of innate immunity with implications for a role in intestinal homeostasis. PLoS One 8:e53876.

Chigerwe, M., D. M. Coons, and J. V. Hagey. 2012. Comparison of colostrum feeding by nipple bottle versus oroesophageal tubing in Holstein dairy bull calves. J. Am. Vet. Med. Assoc. 241:104-109.

Conneely, M., D. P. Berry, J. P. Murphy, I. Lorenz, M. L. Doherty, and E. Kennedy. 2014. Effect of feeding colostrum at different volumes and subsequent number of transition milk feeds on the serum immunoglobulin $\mathrm{G}$ concentration and health status of dairy calves. J. Dairy Sci. 97:6991-7000. 
Curtasu, M. V., P. K. Theil, and M. S. Hedemann. 2016. Metabolomic profiles of colostrum and milk from lactating sows. J. Anim. Sci. 94:272-275.

D’Antona, G., M. Ragni, A. Cardile, L. Tedesco, M. Dossena, F. Bruttini, F. Caliaro, G. Corsetti, R. Bottinelli, M. O. Carruba, A Valerio, and E. Nisoli. 2010. Branched-chain amino acid supplementation promotes survival and supports cardiac and skeletal muscle mitochondrial biogenesis in middle-aged mice. Cell Metab. 12:362-372.

da Silva, R. P., K. B. Kelly, E. D. Lewis, K. A. Leonard, S. Goruk, J. M. Curtis, D. F. Vine, S. D. Proctor, C. J. Field, and R. L. Jacobs. 2015. Choline deficiency impairs intestinal lipid metabolism in the lactating rat. J. Nutr. Biochem. 26:1077-1083.

Danielsen, M., L. J. Pedersen, and E. Bendixen. 2011. An in vivo characterization of colostrum protein uptake in porcine gut during early lactation. J. Proteomics 74:101-109.

Dividich, J. L., J. Marion, and F. Thomas. 2007. Energy and nitrogen utilisation of sow colostrum and milk by the piglet. Can. J. Anim. Sci. 87:571-577.

Ghaffari, M. H., J. A. R. MacPherson, H. Berends, and M. A. Steele. 2017. Diurnal variation of NMR based blood metabolites in calves fed a high plane of milk replacer: a pilot study. BMC Vet. Res. $13: 271$

Girard, J. 1990. Metabolic adaptations to change of nutrition at birth. Biol. Neonate 58:3-15.

Gopal, P. K., and H. S. Gill. 2000. Oligosaccharides and glycoconjugates in bovine milk and colostrum. Br. J. Nutr. 84:S69-S74.

Hammon, H., and J. W. Blum. 1997. The somatotropic axis in neonatal calves can be modulated by nutrition, growth hormone, and Long-R3-IGF-I. Am. J. Physiol. 273:E130-E138.

Hammon, H. M., and J. W. Blum. 1999. Free amino acids in plasma of neonatal calves are influenced by feeding colostrum for different durations or by feeding only milk replacer. J. Anim. Physiol. Anim. Nutr. (Berl.) 82:193-204.

Hammon, H. M., J. Steinhoff-Wagner, J. Flor, U. Schönhusen, and C. C. Metges. 2013. Lactation biology symposium: Role of colostrum and colostrum components on glucose metabolism in neonatal calves. J. Anim. Sci. 91:685-695.

Hammon, H. M., I. A. Zanker, and J. W. Blum. 2000. Delayed colostrum feeding affects IGF-I and insulin plasma concentrations in neonatal calves. J. Dairy Sci. 83:85-92.

Hollenbeck, C. B. 2012. An introduction to the nutrition and metabolism of choline. Cent. Nerv. Syst. Agents Med. Chem. 12:100-113.

Ilcol, Y. O., R. Ozbek, E. Hamurtekin, and I. H. Ulus. 2005. Choline status in newborns, infants, children, breast-feeding women, breast-fed infants and human breast milk. J. Nutr. Biochem. 16:489-499.

Kamada, H., I. Nonaka, Y. Ueda, and M. Murai. 2007. Selenium addition to colostrum increases immunoglobulin $\mathrm{G}$ absorption by newborn calves. J. Dairy Sci. 90:5665-5670.

Kelly, D., and A. G. P. Coutts. 2000. Development of digestive and immunological function in neonates: Role of early nutrition. Livest. Prod. Sci. 66:161-167.

Klein, M. S., N. Buttchereit, S. P. Miemczyk, A. K. Immervoll, C. Louis, S. Wiedemann, W. Junge, G. Thaller, P. J. Oefner, and W. Gronwald. 2012. NMR metabolomic analysis of dairy cows reveals milk glycerophosphocholine to phosphocholine ratio as prognostic biomarker for risk of ketosis. J. Proteome Res. 11:1373-1381.

Liang, G., N. Malmuthuge, T. B. McFadden, H. Bao, P. J. Griebel, P. Stothard, and L. L. Guan. 2014. Potential regulatory role of microRNAs in the development of bovine gastrointestinal tract during early life. PLoS One 9:e92592.

Lin, C., D. C. Mahan, G. Wu, and S. W. Kim. 2009. Protein digestibility of porcine colostrum by neonatal pigs. Livest. Sci. 121:182-186.

Lobley, G. E., S. O. Hoskin, and C. J. McNeil. 2001. Glutamine in animal science and production. J. Nutr. 131:2525S-2531S.

McGrath, B. A., P. F. Fox, P. L. H. McSweeney, and A. L. Kelly. 2016. Composition and properties of bovine colostrum: A review. Dairy Sci. Technol. 96:133-158.
Michel, V., Z. Yuan, S. Ramsubir, and M. Bakovic. 2006. Choline transport for phospholipid synthesis. Exp. Biol. Med. (Maywood) 231:490-504.

Miyazaki, T., K. Okada, T. Yamashita, and M. Miyazaki. 2017. Twodimensional gas chromatography time-of-flight mass spectrometrybased serum metabolic fingerprints of neonatal calves before and after first colostrum ingestion. J. Dairy Sci. 100:4354-4364.

Nakamura, I., K. Ochiai, Y. Imai, F. Moriyasu, and M. Imawari. 2007. Restoration of innate host defense responses by oral supplementation of branched-chain amino acids in decompensated cirrhotic patients. Hepatol. Res. 37:1062-1067.

Nishitani, S., T. Matsumura, S. Fujitani, I. Sonaka, Y. Miura, and K. Yagasaki. 2002. Leucine promotes glucose uptake in skeletal muscles of rats. Biochem. Biophys. Res. Commun. 299:693-696.

Ontsouka, E. C., B. Korczak, H. M. Hammon, and J. W. Blum. 2004b. Real-time PCR quantification of bovine lactase mRNA: localization in the gastrointestinal tract of milk-fed calves. J. Dairy Sci. 87:4230-4237.

Ontsouka, E. C., C. Philipona, H. M. Hammon, and J. W. Blum. 2004a. Abundance of mRNA encoding for components of the somatotropic axis and insulin receptor in different layers of the jejunum and ileum of neonatal calves. J. Anim. Sci. 82:3181-3188.

Osaka, I., Y. Matsui, and F. Terada. 2014. Effect of the mass of immunoglobulin $(\mathrm{Ig}) \mathrm{G}$ intake and age at first colostrum feeding on serum IgG concentration in Holstein calves. J. Dairy Sci. 97:66086612

Palma, M., L. E. Hernández-Castellano, N. Castro, A. Arguëllo, J. Capote, M. Matzapetakis, and A. M. de Almeida. 2016. NMRmetabolomics profiling of mammary gland secretory tissue and milk serum in two goat breeds with different levels of tolerance to seasonal weight loss. Mol. Biosyst. 12:2094-2107.

Phillips, J. W., M. E. Jones, and M. N. Berry. 2002. Implications of the simultaneous occurrence of hepatic glycolysis from glucose and gluconeogenesis from glycerol. Eur. J. Biochem. 269:792-797.

Posho, L., B. Darcy-Vrillon, F. Blachier, and P. H. Duée. 1994. The contribution of glucose and glutamine to energy metabolism in newborn pig enterocytes. J. Nutr. Biochem. 5:284-290.

Putri, S. P., S. Yamamoto, H. Tsugawa, and E. Fukusaki. 2013. Current metabolomics: Technological advances. J. Biosci. Bioeng. 116:9-16.

Quigley, J. 2004. The role of oral immunoglobulins in systemic and intestinal immunity of neonatal calves. Diamond V Mills, Cedar Rapid, IA

Ridgway, N. D. 2013. The role of phosphatidylcholine and choline metabolites to cell proliferation and survival. Crit. Rev. Biochem. Mol. Biol. 48:20-38.

Roth, E., R. Oehler, N. Manhart, R. Exner, B. Wessner, E. Strasser, and A. Spittler. 2002. Regulative potential of glutamine-relation to glutathione metabolism. Nutrition 18:217-221.

Scharrer, E., and B. Grenacher. 2000. Na(+)-dependent transport of D-xylose by bovine intestinal brush border membrane vesicles (BBMV) is inhibited by various pentoses and hexoses. J. Vet. Med. A Physiol. Pathol. Clin. Med. 47:617-626.

Scow, R. O.. E. J. Blanchette-Mackie, and L. C. Smith. 1980. Transport of lipid across capillary endothelium. Fed. Proc. 39:2610-2617.

Spevacek, A. R., J. T. Smilowitz, E. L. Chin, M. A. Underwood, J. B German, and C. M. Slupsky. 2015. Infant maturity at birth reveals minor differences in the maternal milk metabolome in the first month of lactation. J. Nutr. 145:1698-1708.

Steinhoff-Wagner, J., S. Görs, P. Junghans, R. M. Bruckmaier, E. Kanitz, C. C. Metges, and H. M. Hammon. 2011a. Intestinal glucose absorption but not endogenous glucose production differs between colostrum- and formula-fed neonatal calves. J. Nutr. 141:48-55.

Steinhoff-Wagner, J., S. Görs, P. Junghans, R. M. Bruckmaier, E. Kanitz, C. C. Metges, and H. M. Hammon. 2011b. Maturation of endogenous glucose production in preterm and term calves. J. Dairy Sci. 94:5111-5123.

Steinhoff-Wagner, J., R. Zitnan, U. Schonhusen, H. Pfannkuche, M. Hudakova, C. C. Metges, and H. M. Hammon. 2014. Diet effects 
on glucose absorption in the small intestine of neonatal calves: importance of intestinal mucosal growth, lactase activity, and glucose transporters. J. Dairy Sci. 97:6358-6369.

Stott, G. H., D. B. Marx, B. E. Menefee, and G. T. Nightengale. 1979a. Colostral immunoglobulin transfer in calves II. The rate of absorption. J. Dairy Sci. 62:1766-1773.

Stott, G. H., D. B. Marx, B. E. Menefee, and G. T. Nightengale. 1979b. Colostral immunoglobulin transfer in calves. III. Amount of absorption. J. Dairy Sci. 62:1902-1907.

Sundekilde, U. K., P. D. Frederiksen, M. R. Clausen, L. B. Larsen, and H. C. Bertram. 2011. Relationship between the metabolite profile and technological properties of bovine milk from two dairy breeds elucidated by NMR-based metabolomics. J. Agric. Food Chem. 59:7360-7367.

Sundekilde, U. K., L. B. Larsen, and H. C. Bertram. 2013. NMR-based milk metabolomics. Metabolites 3:204-222.

Wu, J., M. Domellöf, A. M. Zivkovic, G. Larsson, A. Öhman, and M. L. Nording. 2016. NMR-based metabolite profiling of human milk: A pilot study of methods for investigating compositional changes during lactation. Biochem. Biophys. Res. Commun. 469:626-632.

Zanker, I. A., H. M. Hammon, and J. W. Blum. 2000. Plasma amino acid pattern during the first month of life in calves fed the first colostrum at $0-2 \mathrm{~h}$ or at $24-25 \mathrm{~h}$ after birth. J. Vet. Med. A Physiol. Pathol. Clin. Med. 47:107-121. 